\title{
Article
}

\section{On certain subclasses of $p$-valent functions with negative coefficients defined by a generalized differential operator}

\author{
Bitrus Sambo $^{1, *}$ and Gideon Benjamin Meller ${ }^{1}$
}

1 Department of Mathematics, Gombe State University, P.M.B.127, Gombe, Nigeria.; bitrussambo3@gmail.com(B.S); gmeller050@gmail.com(G.B.M)

* Correspondence: bitrussambo3@gmail.com

Received: 01 August 2019; Accepted: 03 September 2019; Published: 28 September 2019.

\begin{abstract}
In this article, we introduce new subclasses of normalized analytic functions in the unit disk $U$, defined by a generalized Raducanu-Orhan differential Operator. Various results are driven including coefficient inequalities, growth and distortion theorem, closure property, $\delta$-neighborhoods, extreme points, radii of close-to-convexity, starlikeness and convexity for these subclasses.
\end{abstract}

Keywords: Multivalent functions, Raducanu-Orhan differential operator, extreme points, coefficient inequality, closure properties.

MSC: 30C45, 30C50, 30C55.

\section{Introduction}

$\mathbf{L}$

et $\mathcal{A}$ denote the class of all functions of the form

$$
f(z)=z+\sum_{k=2}^{\infty} a_{k} z^{k}
$$

which are analytic in the open unit disk $U=\{z:|z|<1\}$.

For a function $f \in \mathcal{A}$, Raducanu and Orhan [1] introduced the following operator:

$$
\begin{gathered}
D_{\alpha v}^{0} f(z)=f(z) \\
D_{\alpha v}^{1} f(z)=\alpha v z^{2} f^{\prime \prime}(z)+(\alpha-v) z f^{\prime}(z)+(1-\alpha+v) f(z) \\
D_{\alpha v}^{n} f(z)=D_{\alpha v}\left(D_{\alpha v}^{n-1} f(z)\right),(0 \leq v \leq \alpha \leq 1, n \in N) .
\end{gathered}
$$

If $f$ is given by (1), then from the definition of the operator $D_{\alpha v}^{n} f$, the Equation (2) can be rewritten as:

$$
D_{\alpha v}^{n} f(z)=z+\sum_{k=2}^{\infty}[1+(\alpha v k+\alpha-v)(k-1)]^{n} a_{k} z^{k}
$$

where $\left(n \in N_{0}=N \cup\{0\}\right)$.

Remark 1. 1. When $\alpha=1, v=0$, we get the Sălăgean differential operator introduced by Sălăgean in [2].

2. When $v=0$, we obtain differential operator defined by Al-Oboudi in [3].

Let $\mathcal{A}_{p}$ denote the class of functions of the form

$$
f(z)=z^{p}+\sum_{k=p+1}^{\infty} a_{k} z^{k}, \quad(p=1,2,3, \ldots)
$$


which are analytic and p-valent in the open unit disk $U=\{z:|z|<1\}$. We can write the following equalities for the functions $f \in \mathcal{A}_{p}$ :

$$
\begin{gathered}
D_{\alpha \nu}^{0, p} f(z)=f(z) \\
D_{\alpha \nu}^{1, p} f(z)=\frac{\alpha v}{p} z^{2} f^{\prime \prime}(z)+\frac{1}{p}[(1-p) \alpha v+\alpha-v] z f^{\prime}(z)+(1-\alpha+v) f(z) \\
D_{\alpha \nu}^{n, p} f(z)=D_{\alpha v}\left(D_{\alpha v}^{n-1} f(z)\right), \quad(n \in N=1,2,3, \ldots)
\end{gathered}
$$

If $f$ is given by Equation (4), then from Equation (5) and Equation (6), we see that

$$
D_{\alpha \nu}^{n, p} f(z)=z^{p}+\sum_{k=p+1}^{\infty}\left[1+(\alpha v k+\alpha-v)\left(\frac{k}{p}-1\right)\right]^{n} a_{k} z^{k}, \quad\left(n \in N_{0}=N \cup\{0\}, p \in N=1,2,3, \ldots\right) .
$$

Remark 2. 1. If $v=0, D_{\alpha \nu}^{n, p} f=D_{\alpha, p}^{n} f$ defined by Bulut in [4]

2. If $p=1, D_{\alpha v}^{n, p} f=D_{\alpha v}^{n} f$ introduced by Raducanu and Orhan in [1]

3. If $p=1, \alpha=1, v=0, D_{\alpha v}^{n, p} f=D^{n} f$ defined by Sălăgean in [2]

4. If $p=1, v=0, D_{\alpha v}^{n, p} f=D_{\alpha}^{n} f$ defined by Al-Oboudi in [3].

Let $\mathcal{T}_{p}$ denote the subclass of $\mathcal{A}_{p}$ consisting of functions of the form

$$
f(z)=z^{p}-\sum_{k=p+1}^{\infty} a_{k} z^{k}, \quad\left(a_{k} \geq 0, p=1,2,3, \ldots\right)
$$

If $f$ is given by Equation (8), then from Equation (5) and Equation (6), we get

$$
D_{\alpha v}^{n, p} f(z)=z^{p}-\sum_{k=p+1}^{\infty}\left[1+(\alpha v k+\alpha-v)\left(\frac{k}{p}-1\right)\right]^{n} a_{k} z^{k}, \quad\left(n \in N_{0}\right)
$$

Definition 1. A function $f \in \mathcal{T}_{p}$ is in the class, $S_{p}^{n}(\vartheta, \beta, \gamma, \varphi)$ if and only if

$$
\left|\frac{\left(D_{\alpha \nu}^{n, p} f(z)\right)^{\prime}-p z^{p-1}}{\vartheta\left(D_{\alpha \nu}^{n, p} f(z)\right)^{\prime}+(\beta-\gamma)}\right|<\varphi,\left(z \in U, n \in N_{0}\right)
$$

for $0 \leq v \leq \alpha \leq 1,0 \leq \vartheta<1,0 \leq \gamma<1,0<\beta \leq 1,0<\varphi<1, p \in N, D_{\alpha v}^{n, p} f(z)$ as in (9).

In this paper, basic properties of the class $S_{p}^{n}(\vartheta, \beta, \gamma, \varphi)$ are studied such as: coefficient inequalities, growth and distortion theorem, closure property, $\delta$-neighborhoods, extreme points, radii of close-to-convexity, starlikeness and convexity for these subclasses.

Remark 3. If $v=0, \vartheta=\alpha, \varphi=\mu$, the class $S_{p}^{n}(\vartheta, \beta, \gamma, \varphi)$ reduces to the class $R_{p}^{n}(\alpha, \beta, \gamma, \mu)$ investigated by Bulut [4]

Definition 2. A function $f \in \mathcal{T}_{p}$ is in the class $S_{p}^{n,\left(\delta_{0}\right)}(\vartheta, \beta, \gamma, \varphi)$, if there exists a function $g(z) \in S_{p}^{n}(\vartheta, \beta, \gamma, \varphi)$ such that

$$
\left|\frac{f(z)}{g(z)}-1\right|<1-\delta_{0} \ldots\left(z \in U, 0 \leq \delta_{0}<1\right)
$$

for $0 \leq \vartheta<1,0 \leq \gamma<1,0<\beta \leq 1,0<\varphi<1$.

Definition 3. For a function $f \in \mathcal{T}_{p}, \delta \geq 0, \delta$-neighborhood of $f$ is defined as:

$$
N_{\delta}^{p}(f, g)=\left\{g: g=z^{p}-\sum_{k=p+1}^{\infty} b_{k} z^{k} \in \mathcal{T}_{p} \text { and } \sum_{k=p+1}^{\infty} k\left|a_{k}-b_{k}\right| \leq \delta\right\},
$$


in particular, for a function $h \in \mathcal{T}_{p}$, given by $h(z)=z^{p}(p \in N)$, we immediately have

$$
N_{\delta}^{p}(h, g)=\left\{g: g=z^{p}-\sum_{k=p+1}^{\infty} b_{k} z^{k} \in \mathcal{T}_{p} \text {, and } \sum_{k=p+1}^{\infty} k\left|b_{k}\right| \leq \delta\right\} .
$$

The concept of neighborhoods was first introduced by Goodman [5] and generalized by Ruschewey [6] and Altintas [7] (see also [8,9].

\section{Coefficient inequalities}

Theorem 4. A function $f \in \mathcal{T}_{p}$ is in the class $S_{p}^{n}(\vartheta, \beta, \gamma, \varphi)$ if and only if

$$
\sum_{k=p+1}^{\infty} k\left[1+(\alpha v k+\alpha-v)\left(\frac{k}{p}-1\right)\right]^{n}(1+\varphi \vartheta) a_{k} \leq \varphi(\vartheta p+\beta-\gamma)
$$

for $0 \leq v \leq \alpha \leq 1,0 \leq \vartheta<1,0 \leq \gamma<1,0<\beta \leq 1,0<\varphi<1, n \in N_{0}, p \in N$. Furthermore, the result is sharp for the function given as

$$
f(z)=z^{p}-\frac{\varphi(\vartheta p+\beta-\gamma)}{k\left[1+(\alpha v k+\alpha-v)\left(\frac{k}{p}-1\right)\right]^{n}(1+\varphi \vartheta)} a_{k}(k \geq p+1) .
$$

Proof. Suppose that $f \in S_{p}^{n}(\vartheta, \beta, \gamma, \varphi)$, then from inequality (10), we have

$$
\begin{aligned}
\left|\frac{\left(D_{\alpha \nu}^{n, p} f(z)\right)^{\prime}-p z^{p-1}}{\vartheta\left(D_{\alpha \nu}^{n, p} f(z)\right)^{\prime}+(\beta-\gamma)}\right| & =\left|\frac{p z^{p-1}-\sum_{k=p+1}^{\infty} k\left[1+(\alpha v k+\alpha-v)\left(\frac{k}{p}-1\right)\right]^{n} a_{k} z^{k-1}-p z^{p-1}}{\vartheta\left(p z^{p-1}-\sum_{k=p+1}^{\infty} k\left[1+(\alpha v k+\alpha-v)\left(\frac{k}{p}-1\right)\right]^{n} a_{k} z^{k-1}\right)+(\beta-\gamma)}\right| \\
& =\left|\frac{\sum_{k=p+1}^{\infty} k\left[1+(\alpha v k+\alpha-v)\left(\frac{k}{p}-1\right)\right]^{n} a_{k} z^{k-1}}{\vartheta\left(p z^{p-1}-\sum_{k=p+1}^{\infty} k\left[1+(\alpha v k+\alpha-v)\left(\frac{k}{p}-1\right)\right]^{n} a_{k} z^{k-1}\right)+(\beta-\gamma)}\right| \\
& <\varphi,\left(z \in U, n \in N_{0}\right)
\end{aligned}
$$

it is well known that $\Re z \leq|z|$, therefore, we obtain

$$
\Re\left\{\frac{\sum_{K=p+1}^{\infty} k\left[1+(\alpha v k+\alpha-v)\left(\frac{k}{p}-1\right)\right]^{n} a_{k} z^{k-1}}{\vartheta\left(p z^{p-1}-\sum_{k=p+1}^{\infty} k\left[1+(\alpha v k+\alpha-v)\left(\frac{k}{p}-1\right)\right]^{n} a_{k} z^{k-1}\right)+(\beta-\gamma)}\right\}<\varphi .
$$

If we choose $z$ real and let $z \rightarrow 1^{-}$, then we get

$$
\sum_{K=p+1}^{\infty} k\left[1+(\alpha v k+\alpha-v)\left(\frac{k}{p}-1\right)\right]^{n} a_{k} \leq \varphi\left\{\vartheta\left(p-\sum_{k=p+1}^{\infty} k\left[1+(\alpha v k+\alpha-v)\left(\frac{k}{p}-1\right)\right]^{n} a_{k}\right)+(\beta-\gamma)\right\}
$$

which is precisely the assertion (13).

On contrary, suppose that the inequality (13) hold true and let $z \in \delta U=\{z \in C:|z|=1\}$. Then, from (10), we have

$$
\begin{aligned}
& \left|\left(D_{\alpha v}^{n, p} f(z)\right)^{\prime}-p z^{p-1}\right|-\varphi\left|\vartheta\left(D_{\alpha v}^{n, p} f(z)\right)^{\prime}+(\beta-\gamma)\right| \leq \sum_{k=p+1}^{\infty} k\left[1+(\alpha v k+\alpha-v)\left(\frac{k}{p}-1\right)\right]^{n} a_{k}|z|^{k-1} \\
& -\varphi(\vartheta p+\beta-\gamma)+\varphi \vartheta \sum_{k=p+1}^{\infty} k\left[1+(\alpha v k+\alpha-v)\left(\frac{k}{p}-1\right)\right]^{n} a_{k}|z|^{k-1} \\
& =\sum_{k=p+1}^{\infty} k\left[1+(\alpha v k+\alpha-v)\left(\frac{k}{p}-1\right)\right]^{n} a_{k}|z|^{k-1}(1+\varphi \vartheta) a_{k}-\varphi(\vartheta p+\beta-\gamma) \leq 0 .
\end{aligned}
$$


By maximum modulus theorem, we have $f \in S_{p}^{n}(\vartheta, \beta, \gamma, \varphi)$.

Corollary 5. If $f \in S_{p}^{n}(\vartheta, \beta, \gamma, \varphi)$, then $a_{p+1} \leq \frac{\varphi(\vartheta p+\beta-\gamma)}{(p+1)\left[1+(\alpha v(p+1)+\alpha-v)\left(\frac{1}{p}\right)\right]^{n}(1+\varphi \vartheta)}$.

\section{Growth and distortion theorem}

Theorem 6. For each $f(z) \in S_{p}^{n}(\vartheta, \beta, \gamma, \varphi)$, we have $|z|^{p}-\frac{\varphi(\vartheta p+\beta-\gamma)}{\left[1+(\alpha v(p+1)+\alpha-v)\left(\frac{1}{p}\right)\right]^{n}(1+\varphi \vartheta)(p+1)}|z|^{p+1} \leq|f(z)| \leq|z|^{p}+\frac{\varphi(\vartheta p+\beta-\gamma)}{\left[1+(\alpha v(p+1)+\alpha-v)\left(\frac{1}{p}\right)\right]^{n}(1+\varphi \vartheta)(p+1)}|z|^{p+1}$.

Proof. Let $f(z) \in S_{p}^{n}(\vartheta, \beta, \gamma, \varphi), z \in U$, the bound on $f(z)$ is given by

$$
|f(z)| \leq|z|^{p}+|z|^{p+1} \sum_{k=p+1}^{\infty} a_{k}, z \in U
$$

from Theorem 4, we have

$$
\sum_{k=p+1}^{\infty} a_{k} \leq \frac{\varphi(\vartheta p+\beta-\gamma)}{(p+1)\left[1+(\alpha v(p+1)+\alpha-v)\left(\frac{1}{p}\right)\right]^{n}(1+\varphi \vartheta)},
$$

by using (15) in (14), we obtain

$$
|f(z)| \leq|z|^{p}+\frac{\varphi(\vartheta p+\beta-\gamma)}{(p+1)\left[1+(\alpha v(p+1)+\alpha-v)\left(\frac{1}{p}\right)\right]^{n}(1+\varphi \vartheta)}|z|^{p+1},
$$

again using (15), we have

$$
|f(z)| \geq|z|^{p}-\frac{\varphi(\vartheta p+\beta-\gamma)}{(p+1)\left[1+(\alpha v(p+1)+\alpha-v)\left(\frac{1}{p}\right)\right]^{n}(1+\varphi \vartheta)}|z|^{p+1} .
$$

Consequently, combining (16) and (17) we obtain the desired result.

Theorem 7. For each $f(z) \in S_{p}^{n}(\vartheta, \beta, \gamma, \varphi)$, we have

$$
p|z|^{p-1}-\frac{\varphi(\vartheta p+\beta-\gamma)}{\left[1+(\alpha v(p+1)+\alpha-v)\left(\frac{1}{p}\right)\right]^{n}(1+\varphi \vartheta)}|z|^{p} \leq\left|f^{\prime}(z)\right| \leq p|z|^{p-1}+\frac{\varphi(\vartheta p+\beta-\gamma)}{\left[1+(\alpha v(p+1)+\alpha-v)\left(\frac{1}{p}\right)\right]^{n}(1+\varphi \vartheta)}|z|^{p} .
$$

Proof. Let $f(z) \in S_{p}^{n}(\vartheta, \beta, \gamma, \varphi), z \in U$, the bound on the derivative of $f(z)$ is given by

$$
\left|f^{\prime}(z)\right| \leq p|z|^{p-1}+(p+1)|z|^{p} \sum_{k=p+1}^{\infty} a_{k}, z \in U,
$$

and, in the same way as above, we get our desired result.

\section{Closure properties}

Theorem 8. Let the functions

$$
\begin{array}{ll}
f(z)=z^{p}-\sum_{k=p+1}^{\infty} a_{k} z^{k}, & \left(a_{k} \geq 0\right) \\
g(z)=z^{p}-\sum_{k=p+1}^{\infty} b_{k} z^{k}, & \left(b_{k} \geq 0\right)
\end{array}
$$


be in the class $S_{p}^{n}(\vartheta, \beta, \gamma, \varphi)$. Then for $0 \leq \lambda \leq 1$, the function $h$ is defined as

$$
h(z)=(1-\lambda) f(z)+\lambda g(z)=z^{p}-\sum_{k=p+1}^{\infty} c_{k} z^{k}
$$

where $c_{k}:=(1-\lambda) a_{k}+\lambda b_{k} \geq 0$, is also in $S_{p}^{n}(\vartheta, \beta, \gamma, \varphi)$.

Proof. Suppose that each of the functions $f$ and $g$ is in the class $S_{p}^{n}(\vartheta, \beta, \gamma, \varphi)$. Then making use of inequality (13), we have

$$
\begin{aligned}
& \sum_{k=p+1}^{\infty} k\left[1+(\alpha v k+\alpha-v)\left(\frac{k}{p}-1\right)\right]^{n}(1+\varphi \vartheta) c_{k} \\
& =(1-\lambda) \sum_{k=p+1}^{\infty} k\left[1+(\alpha v k+\alpha-v)\left(\frac{k}{p}-1\right)\right]^{n}(1+\varphi \vartheta) a_{k} \\
& +\lambda \sum_{k=p+1}^{\infty} k\left[1+(\alpha v k+\alpha-v)\left(\frac{k}{p}-1\right)\right]^{n}(1+\varphi \vartheta) b_{k} \\
& \leq(1-\lambda) \varphi(\vartheta p+\beta-\gamma)+\lambda \varphi(\vartheta p+\beta-\gamma) \\
& =\varphi(\vartheta p+\beta-\gamma)
\end{aligned}
$$

which completes the proof.

\section{5. $\delta$-Neighborhoods}

Theorem 9. If

$$
\delta:=\frac{\varphi(\vartheta p+\beta-\gamma)}{\left[1+(\alpha v(p+1)+\alpha-v)\left(\frac{1}{p}\right)\right]^{n}(1+\varphi \vartheta)},
$$

then $S_{p}^{n}(\vartheta, \beta, \gamma, \varphi) \subset N_{\delta}^{p}(h, g)$.

Proof. For a function $f(z) \in S_{p}^{n}(\vartheta, \beta, \gamma, \varphi)$ of the form (8), Theorem 4 immediately yields

$$
(p+1)\left[1+(\alpha v(p+1)+\alpha-v)\left(\frac{1}{p}\right)\right]^{n}(1+\varphi \vartheta) \sum_{k=p+1}^{\infty} a_{k} \leq \varphi(\vartheta p+\beta-\gamma),
$$

therefore,

$$
\sum_{k=p+1}^{\infty} a_{k} \leq \frac{\varphi(\vartheta p+\beta-\gamma)}{(p+1)\left[1+(\alpha v(p+1)+\alpha-v)\left(\frac{1}{p}\right)\right]^{n}(1+\varphi \vartheta)} .
$$

On the other hand, we also find from (13) that

$$
\sum_{k=p+1}^{\infty} k a_{k} \leq \frac{\varphi(\vartheta p+\beta-\gamma)}{\left[1+(\alpha v(p+1)+\alpha-v)\left(\frac{1}{p}\right)\right]^{n}(1+\varphi \vartheta)},
$$

that is

$$
\sum_{k=p+1}^{\infty} k a_{k} \leq \frac{\varphi(\vartheta p+\beta-\gamma)}{\left[1+(\alpha v(p+1)+\alpha-v)\left(\frac{1}{p}\right)\right]^{n}(1+\varphi \vartheta)}:=\delta,
$$

which completes the proof. 
Theorem 10. If $g(z) \in S_{p}^{n}(\vartheta, \beta, \gamma, \varphi)$ and

$$
\delta_{0}=1-\frac{\delta}{p+1} \frac{(p+1)\left[1+(\alpha v(p+1)+\alpha-v)\left(\frac{1}{p}\right)\right]^{n}(1+\varphi \vartheta)}{(p+1)\left[1+(\alpha v(p+1)+\alpha-v)\left(\frac{1}{p}\right)\right]^{n}(1+\varphi \vartheta)-\varphi(\vartheta p+\beta-\gamma)},
$$

then $N_{\delta}^{p}(f, g) \subset S_{p}^{n,\left(\delta_{0}\right)}(\vartheta, \beta, \gamma, \varphi)$.

Proof. Suppose that $f \in N_{\delta}^{p}(f, g)$, then by Definition 3, we have

$$
\sum_{k=p+1}^{\infty} k\left|a_{k}-b_{k}\right| \leq \delta
$$

which readily implies the coefficient inequality given by

$$
\sum_{k=p+1}^{\infty}\left|a_{k}-b_{k}\right| \leq \frac{\delta}{p+1}(p \in N) .
$$

Next, since $g \in S_{p}^{n}(\vartheta, \beta, \gamma, \varphi)$, we have from inequality (13) that

$$
\sum_{k=p+1}^{\infty} b_{k} \leq \frac{\varphi(\vartheta p+\beta-\gamma)}{(p+1)\left[1+(\alpha v(p+1)+\alpha-v)\left(\frac{1}{p}\right)\right]^{n}(1+\varphi \vartheta)}
$$

so from the definition of the class, we have

$$
\begin{aligned}
\left|\frac{f(z)}{g(z)}-1\right| & <\frac{\sum_{k=p+1}^{\infty}\left|a_{k}-b_{k}\right|}{1-\sum_{k=p+1}^{\infty} b_{k}} \\
& \leq \frac{\delta}{p+1} \frac{(p+1)\left[1+(\alpha v(p+1)+\alpha-v)\left(\frac{1}{p}\right)\right]^{n}(1+\varphi \vartheta)}{(p+1)\left[1+(\alpha v(p+1)+\alpha-v)\left(\frac{1}{p}\right)\right]^{n}(1+\varphi \vartheta)-\varphi(\vartheta p+\beta-\gamma)} \\
& =1-\delta_{0},
\end{aligned}
$$

provided that $\delta_{0}$ is given precisely by (22). Thus, by the definition, $f \in S_{p}^{n, \delta_{0}}(\vartheta, \beta, \gamma, \varphi)$ for $\delta_{0}$ given by (22), this completes our proof.

\section{Extreme points}

Theorem 11. If $f_{p}(z)=z^{p}, f_{k}(z)=z^{p}-\frac{\varphi(\vartheta p+\beta-\gamma)}{k\left[1+(\alpha v k+\alpha-v)\left(\frac{k}{p}-1\right)\right]^{n}(1+\varphi \vartheta)} z^{k}(k \geq p+1)$ then, $f \in S_{p}^{n}(\vartheta, \beta, \gamma, \varphi)$ if and only if it can be expressed in the form $f(z)=\lambda_{p} f_{p}(z)+\sum_{k=p+1}^{\infty} \lambda_{k} f_{k}(z)$, where $\lambda_{k} \geq 0$ and $\lambda_{p}=1-\sum_{k=p+1}^{\infty} \lambda_{k}$.

Proof. Assume that $f(z)=\lambda_{p} f_{p}(z)+\sum_{k=p+1}^{\infty} \lambda_{k} f_{k}(z)$, then

$$
\begin{aligned}
f(z) & =\left(1-\sum_{k=p+1}^{\infty} \lambda_{k}\right) z^{p}+\sum_{k=p+1}^{\infty} \lambda_{k}\left\{z^{p}-\frac{\varphi(\vartheta p+\beta-\gamma)}{k\left[1+(\alpha v k+\alpha-v)\left(\frac{k}{p}-1\right)\right]^{n}(1+\varphi \vartheta)} z^{k}\right\} \\
& =z^{p}-\sum_{k=p+1}^{\infty} \lambda_{k}\left\{\frac{\varphi(\vartheta p+\beta-\gamma)}{k\left[1+(\alpha v k+\alpha-v)\left(\frac{k}{p}-1\right)\right]^{n}(1+\varphi \vartheta)} z^{k}\right\} .
\end{aligned}
$$


Thus,

$$
\begin{aligned}
& \sum_{k=p+1}^{\infty} k\left[1+(\alpha v k+\alpha-v)\left(\frac{k}{p}-1\right)\right]^{n}(1+\varphi \vartheta) \lambda_{k} \frac{\varphi(\vartheta p+\beta-\gamma)}{k\left[1+(\alpha v k+\alpha-v)\left(\frac{k}{p}-1\right)\right]^{n}(1+\varphi \vartheta)} \\
& =\varphi(\vartheta p+\beta-\gamma) \sum_{k=p+1}^{\infty} \lambda_{k}=\varphi(\vartheta p+\beta-\gamma)\left(1-\lambda_{p}\right) \leq \varphi(\vartheta p+\beta-\gamma),
\end{aligned}
$$

which shows that $f$ satisfies condition (13) and therefore, $f \in S_{p}^{n}(\vartheta, \beta, \gamma, \varphi)$. Conversely, suppose that $f \in$ $S_{p}^{n}(\vartheta, \beta, \gamma, \varphi)$, since

$$
a_{k} \leq \frac{\varphi(\vartheta p+\beta-\gamma)}{k\left[1+(\alpha v k+\alpha-v)\left(\frac{k}{p}-1\right)\right]^{n}(1+\varphi \vartheta)},(k \geq p+1),
$$

we may set

$$
\lambda_{k}=\frac{k\left[1+(\alpha v k+\alpha-v)\left(\frac{k}{p}-1\right)\right]^{n}(1+\varphi \vartheta)}{\varphi(\vartheta p+\beta-\gamma)} a_{k} \text {, and } \lambda_{p}=1-\sum_{k=p+1}^{\infty} \lambda_{k}
$$

then we obtain from

$$
\begin{aligned}
f(z) & =z^{p}-\sum_{k=p+1}^{\infty} a_{k} z^{k} \\
& =\left(\lambda_{p}+\sum_{k=p+1}^{\infty} \lambda_{k}\right) z^{p}-\sum_{k=p+1}^{\infty} \lambda_{k} \frac{\varphi(\vartheta p+\beta-\gamma)}{k\left[1+(\alpha v k+\alpha-v)\left(\frac{k}{p}-1\right)\right]^{n}(1+\varphi \vartheta)} z^{k} \\
& =\lambda_{p} z^{p}+\sum_{k=p+1}^{\infty} \lambda_{k}\left(z^{p}-\frac{\varphi(\vartheta p+\beta-\gamma)}{k\left[1+(\alpha v k+\alpha-v)\left(\frac{k}{p}-1\right)\right]^{n}(1+\varphi \vartheta)} z^{k}\right) \\
& =\lambda_{p} z^{p}+\sum_{k=p+1}^{\infty} \lambda_{k} f_{k}(z)
\end{aligned}
$$

which completes the proof.

Corollary 12. The extreme points of $S_{p}^{n}(\vartheta, \beta, \gamma, \varphi)$ are given by

$$
f_{p}(z)=z^{p}, f_{k}(z)=z^{p}-\frac{\varphi(\vartheta p+\beta-\gamma)}{k\left[1+(\alpha v k+\alpha-v)\left(\frac{k}{p}-1\right)\right]^{n}(1+\varphi \vartheta)} z^{k}(k \geq p+1)
$$

\section{Radii of close-to-convexity,starlikeness and convexity}

A function $f \in \mathcal{T}_{p}$ is said to be $p$-valently close-to-convex of order $\rho$ if it satisfies

$$
\Re\left\{f^{\prime}(z)\right\}>\rho
$$

for some $\rho(0 \leq \rho<p)$ and for all $z \in U$.

Also, a function $f \in \mathcal{T}_{p}$ is said to be $p$-valently starlike of order $\rho$ if it satisfies

$$
\Re\left\{\frac{z f^{\prime}(z)}{f(z)}\right\}>\rho,
$$

for some $\rho(0 \leq \rho<p)$ and for all $z \in U$.

Further, a function $f \in \mathcal{T}_{p}$ is said to be $p$-valently convex of order $\rho$ if it satisfies

$$
\Re\left\{1+\frac{z f^{\prime \prime}(z)}{f^{\prime}(z)}\right\}>\rho,
$$

for some $\rho(0 \leq \rho<p)$ and for all $z \in U$. 
Theorem 13. If $f \in S_{p}^{n}(\vartheta, \beta, \gamma, \varphi)$ then $f$ is $p$-valently close-to-convex of order $\rho$ in $|z|<r_{1}(\vartheta, \beta, \gamma, \varphi, \rho)$, where

$$
r_{1}(\vartheta, \beta, \gamma, \varphi, \rho)=\inf _{k}\left\{\frac{\left[1+(\alpha v k+\alpha-v)\left(\frac{k}{p}-1\right)\right]^{n}(1+\varphi \vartheta) a_{k}(p-\rho)}{\varphi(\vartheta p+\beta-\gamma)}\right\}^{\frac{1}{k-p}} k \geq p+1
$$

Proof. It is sufficient to show that $\left|\frac{f^{\prime}(z)}{z^{p-1}}-p\right|<p-\rho$. Since $\left|\frac{p z^{p-1}-\sum_{k=p+1}^{\infty} k a_{K} z^{k-1}}{z^{p-1}}-p\right|<p-\rho$, which implies that

$$
\left|\frac{f^{\prime}(z)}{z^{p-1}}-p\right| \leq \sum_{k=p+1}^{\infty} k a_{k}|z|^{k-p}<p-\rho,
$$

implies

$$
\frac{\sum_{k=p+1}^{\infty} k a_{k}|z|^{k-p}}{p-\rho}<1
$$

and by applying the result of Theorem 4, we get

$$
\sum_{k=p+1}^{\infty} a_{k} \leq \frac{\varphi(\vartheta p+\beta-\gamma)}{k\left[1+(\alpha v k+\alpha-v)\left(\frac{k}{p}-1\right)\right]^{n}(1+\varphi \vartheta) a_{k}}
$$

Hence,(23) is true if

$$
\frac{k|z|^{k-p}}{p-\rho} \leq \frac{k\left[1+(\alpha v k+\alpha-v)\left(\frac{k}{p}-1\right)\right]^{n}(1+\varphi \vartheta)}{\varphi(\vartheta p+\beta-\gamma)}
$$

solving (24) for $z$ we obtain

$$
|z| \leq\left\{\frac{\left[1+(\alpha v k+\alpha-v)\left(\frac{k}{p}-1\right)\right]^{n}(1+\varphi \vartheta)(p-\rho)}{\varphi(\vartheta p+\beta-\gamma)}\right\}^{\frac{1}{k-p}}
$$

which completes the proof.

Theorem 14. If $f \in S_{p}^{n}(\vartheta, \beta, \gamma, \varphi)$ then $f$ is $p$-valently starlike of order $\rho$ in $|z|<r_{2}(\vartheta, \beta, \gamma, \varphi, \rho)$, where

$$
r_{2}(\vartheta, \beta, \gamma, \varphi, \rho)=\inf _{k}\left\{\frac{k\left[1+(\alpha v k+\alpha-v)\left(\frac{k}{p}-1\right)\right]^{n}(1+\varphi \vartheta)(p-\rho)}{\varphi(\vartheta p+\beta-\gamma)(k-\rho)}\right\}^{\frac{1}{k-p}} k \geq p+1 .
$$

Proof. In order to prove, it suffices to show that $\left|\frac{z f^{\prime}(z)}{f(z)}-p\right|<p-\rho$.

$$
\begin{aligned}
\left|\frac{z f^{\prime}(z)}{f(z)}-p\right| & =\left|\frac{z f^{\prime}(z)-p f(z)}{f(z)}\right| \\
& =\left|\frac{z\left(p z^{p-1}-\sum_{k=p+1}^{\infty} k a_{k} z^{k-1}\right)-p\left(z^{p}-\sum_{k=p+1}^{\infty} a_{k} z^{k}\right)}{z^{p}-\sum_{k=p+1}^{\infty} a_{k} z^{k}}\right| \\
& =\left|\frac{-\sum_{k=p+1}^{\infty}(k-p) a_{k} z^{k-p}}{1-\sum_{k=p+1}^{\infty} a_{k} z^{k-p}}\right| \leq \frac{\sum_{k=p+1}^{\infty}(k-p) a_{k}|z|^{k-p}}{1-\sum_{k=p+1}^{\infty} a_{k}|z|^{k-p}}<p-\rho,
\end{aligned}
$$


and by using inequality (13), we get

$$
\sum_{k=p+1}^{\infty} a_{k} \leq \frac{\varphi(\vartheta p+\beta-\gamma)}{k\left[1+(\alpha v k+\alpha-v)\left(\frac{k}{p}-1\right)\right]^{n}(1+\varphi \vartheta) a_{k}}
$$

so, (25) holds true if

$$
\frac{(k-\rho)|z|^{k-\rho}}{p-\rho} \leq \frac{k\left[1+(\alpha v k+\alpha-v)\left(\frac{k}{p}-1\right)\right]^{n}(1+\varphi \vartheta)}{\varphi(\vartheta p+\beta-\gamma)},
$$

and then $f$ is starlike of order $\rho$.

Theorem 15. If $f \in S_{p}^{n}(\vartheta, \beta, \gamma, \varphi)$, then $f$ is $p$-valently convex of order $\rho$ in $|z|<r_{3}(\vartheta, \beta, \gamma, \varphi, \rho)$, where

$$
r_{3}(\vartheta, \beta, \gamma, \varphi, \rho)=\inf _{k}\left\{\frac{\left[1+(\alpha v k+\alpha-v)\left(\frac{k}{p}-1\right)\right]^{n}(1+\varphi \vartheta) p(p-\rho)}{\varphi(\vartheta p+\beta-\gamma)(k-\rho)}\right\}^{\frac{1}{k-p}} k \geq p+1 .
$$

Proof. To prove this, it suffices to show that $\left|1+\frac{z f^{\prime \prime}(z)}{f^{\prime}(z)}-p\right|<p-\rho$.

Since

$$
\begin{aligned}
& \left|1+\frac{z f^{\prime \prime}(z)}{f^{\prime}(z)}-p\right|=\left|\frac{f^{\prime}(z)+z f^{\prime \prime}(z)-p f^{\prime}(z)}{f^{\prime}(z)}\right| \\
& =\left|\frac{p z^{p-1}-\sum_{k=p+1}^{\infty} k a_{k} z^{k-1}+z\left(p(p-1) z^{p-2}-\sum_{k=p+1}^{\infty} k(k-1) a_{k} z^{k-2}\right)-p\left(p z^{p-1}-\sum_{k=p+1}^{\infty} k a_{k} z^{k-1}\right)}{p z^{p-1}-\sum_{k=p+1}^{\infty} k a_{k} z^{k-1}}\right|
\end{aligned}
$$

it implies that

$$
\left|1+\frac{z f^{\prime \prime}(z)}{f^{\prime}(z)}-p\right|=\left|\frac{-\sum_{k=p+1}^{\infty} k(k-p) a_{k} z^{k-p}}{p-\sum_{k=p+1}^{\infty} k a_{k} z^{k-p}}\right| \leq \frac{\sum_{k=p+1}^{\infty} k(k-p) a_{k}|z|^{k-p}}{p-\sum_{k=p+1}^{\infty} k a_{k}|z|^{k-p}}<p-\rho
$$

and by applying the result in Theorem 4, we get

$$
\sum_{k=p+1}^{\infty} a_{k} \leq \frac{\varphi(\vartheta p+\beta-\gamma)}{k\left[1+(\alpha v k+\alpha-v)\left(\frac{k}{p}-1\right)\right]^{n}(1+\varphi \vartheta) a_{k}}
$$

so, (26) holds true if

$$
\frac{k(k-\rho)|z|^{k-p}}{p(p-\rho)} \leq \frac{k\left[1+(\alpha v k+\alpha-v)\left(\frac{k}{p}-1\right)\right]^{n}(1+\varphi \vartheta)}{\varphi(\vartheta p+\beta-\gamma)}
$$

and then $f$ is convex of order $\rho$.

Author Contributions: All authors contributed equally to the writing of this paper. All authors read and approved the final manuscript.

Conflicts of Interest: "The authors declare no conflict of interest."

\section{References}

[1] Raducanu, D., \& Orhan, H. (2010). Subclasses of analytic functions defined by a generalized differential operator. International Journal of Mathematical Analysis, 4(1), 1-15.

[2] Salagean, G. S. (1983). Subclasses of univalent functions, Complex analysis-fifth Romanian-Finnish seminar, Part 1 (Bucharest, 1981), 362-372. Lecture Notes in Math, 1013.

[3] Al-Oboudi, F. M. (2004). On univalent functions defined by a generalized Sălăgean. International Journal of Mathematics and Mathematical Sciences, 2004(27), 1429-1436. 
[4] Bulut, S. (2010). On a class of analytic and multivalent functions with negative coefficients defined by Al-Oboudi differential operator. Mathematica, 115.

[5] Goodman, A. W. (1957). Univalent functions and non-Analytic curves. Proceedings of the American Mathematical Society, $8,598-601$.

[6] Ruscheweyh, S. (1981). Neighborhoods of univalent functions. Proceedings of the American Mathematical Society, 81(4), 521-527.

[7] Altintas, O., Irmak, H., \& Srivastava, H. M. (2008). Neighborhoods for certain subclasses of multivalently analytic functions defined by using a differential operator. Computers $\mathcal{E}$ Mathematics with Applications, 55(3), 331-338.

[8] Altintas, O., Özkan, Ö., \& Srivastava, H. M. (2004). Neighborhoods of a certain family of multivalent functions with negative coefficients. Computers \& Mathematics with Applications, 47(10-11), 1667-1672.

[9] Altintas, O., \& Owa, S. (1996). Neighborhoods of certain analytic functions with negative coefficients. International Journal of Mathematics and Mathematical Sciences, 19(4), 797-800.

(C) 2019 by the authors; licensee PSRP, Lahore, Pakistan. This article is an open access article distributed under the terms and conditions of the Creative Commons Attribution (CC-BY) license (http://creativecommons.org/licenses/by/4.0/). 